This item was submitted to Loughborough's Research Repository by the author.

Items in Figshare are protected by copyright, with all rights reserved, unless otherwise indicated.

\title{
A phase feedback based extended space-time block code for enhancement of diversity
}

PLEASE CITE THE PUBLISHED VERSION

PUBLISHER

(c) IEEE

VERSION

VoR (Version of Record)

LICENCE

CC BY-NC-ND 4.0

\section{REPOSITORY RECORD}

Eltayeb, Nasr E.M., Sangarapillai Lambotharan, and Jonathon Chambers. 2019. "A Phase Feedback Based Extended Space-time Block Code for Enhancement of Diversity”. figshare. https://hdl.handle.net/2134/5640. 
This item was submitted to Loughborough's Institutional Repository (https://dspace.lboro.ac.uk/) by the author and is made available under the following Creative Commons Licence conditions.

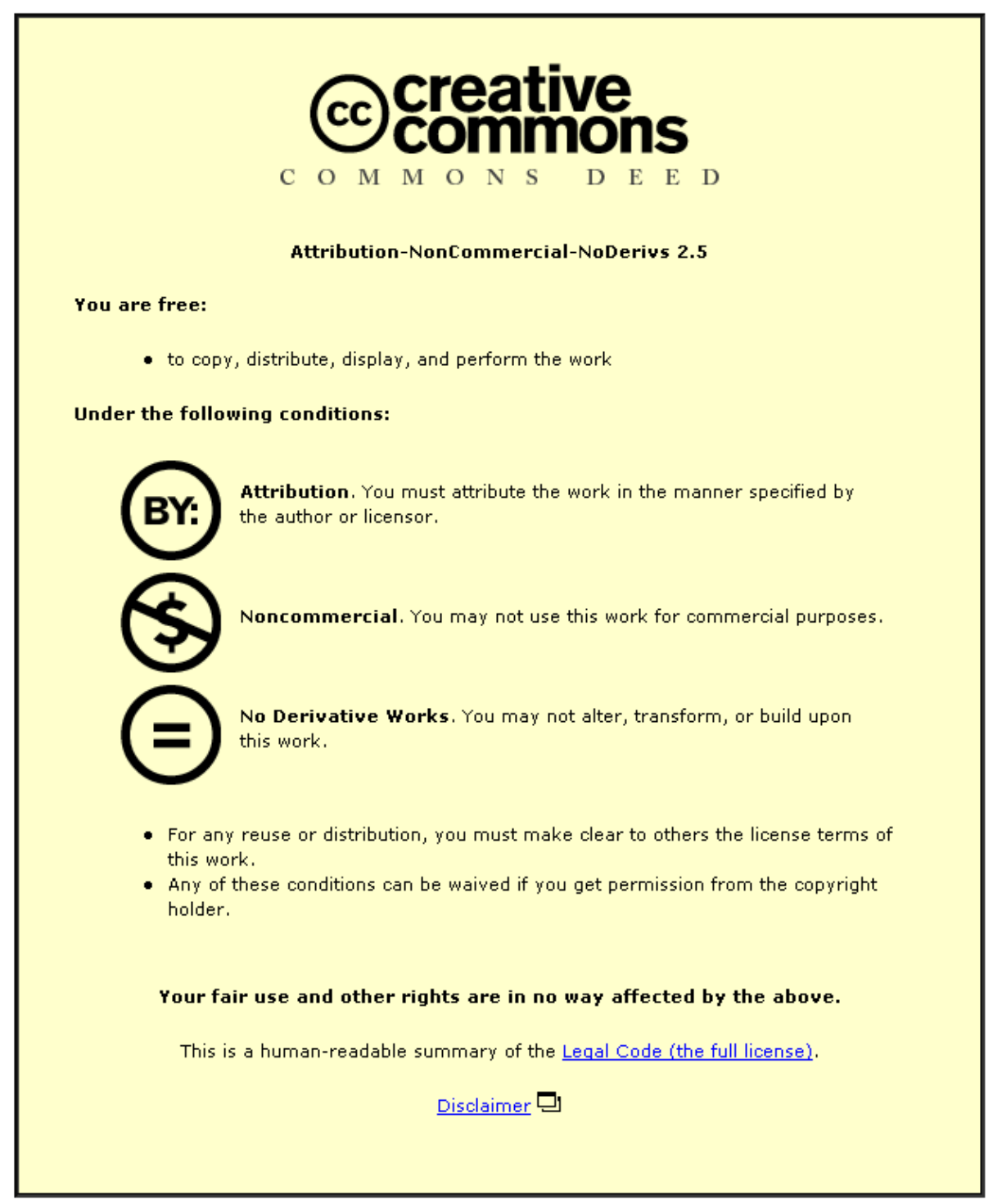

For the full text of this licence, please go to: http://creativecommons.org/licenses/by-nc-nd/2.5/ 


\title{
A Phase Feedback Based Extended Space-Time Block Code for Enhancement of Diversity
}

\author{
N. M. Eltayeb, S. Lambotharan, and J. A. Chambers \\ Centre of Digital Signal Processing, Cardiff University, CF24 3AA, United Kingdom \\ Email: eltayebne, lambotharans, chambersj@cardiff.ac.uk
}

\begin{abstract}
In this paper we propose a generalization of extended orthogonal space-time block codes (EO-STBCs) for MIMO (multi-input/multi-output) channels using four transmit antennas for quasi-static flat fading channels. Since full rate and complex orthogonal space-time block codes (STBCs) do not exist for more than two transmit antennas, we propose a feedback based STBC scheme. In this scheme, phases of certain symbols are rotated according to the feedback from the receiver which is equivalent to rotating the phases of the corresponding channel coefficients. Simulation results show that this rotation phase feedback method achieves a satisfactory performance and outperforms the previous closed-loop space-time block codes, even when the feedback is quantized.
\end{abstract}

Index Terms- Extended orthogonal space-time block codes (EO-STBC), closed-loop phase angle feedback, multi-input/multi-output (MIMO).

\section{INTRODUCTION}

$\mathrm{T}$ ransmit diversity, a form of spatial diversity, is a technique of combating detrimental effects in wireless fading channels. The use of multiple transmit antennas for diversity provides better performance without increasing the bandwidth or transmission power. The Alamouti scheme, one of the most well known transmit diversity techniques, is an orthogonal space-time block code (STBC) [1]. It is the only complex orthogonal STBC that can achieve full diversity gain and rate one. Higher order STBCs [2] have been proposed but either diversity or full code rate must be relaxed. A quasi-orthogonal space-time block code (QO-STBCs) is designed in [3] for four transmit antennas which achieves full code rate at the expense of loss in diversity gain. Results show that full transmission rate is more practical for very low signal-to-noise ratio (SNR) and high bit-error rate (BER); whereas, full diversity is the right candidate for high SNR and low BER. As demonstrates in [4], the loss of diversity in QO-STBCs is due to a coupling term between the estimated symbols.

In the analysis of space-time block codes (STBCs) it is usually assumed that the transmitter has no knowledge about the channel. The channel conditions can however be observed at the receiver. Exploiting channel state information (CSI) at the transmitter through feedback from the receiver, is therefore a good strategy to improve the performance of many physical layer techniques, including STBCs, provided the channel variation is limited.

Research on adapting several closed-loop methods for STBCs has been proposed to attain full code rate and full diversity. In [4], [5], [6], and [12], the phases of the signals transmitted from certain antennas are rotated in a prescribed way in order to make the code orthogonal based upon the information fed back from the receiver, thereby increasing the diversity gain. For a slow fading channel, only two-bit feedback is shown to be sufficient to achieve full diversity. With partial channel information available at the transmitter, beamforming techniques can be combined with space-time block coding to further improve the system performance. Those two technologies can be employed at the transmitter to provide transmit diversity and beamforming gain to increase the SNR of a wireless system. Examples of such systems are found in [9], and [10]. For open-loop wireless systems, Xia et. al. presented another scheme called the constellation rotated method in [11] for QO-STBCs. Full-rate and full diversity are achievable if signal constellations are chosen properly. However, the tradeoff is that the size of the signal constellations increases and decoding becomes more complex.

In this work, we consider a multiple transmitter and single receiver system in the presence of feedback. In particular, we develop a new scheme to maximize the SNR improvement as compared to previous work [8]. The associated simulations show that the proposed method provides a significant improvement in BER performance, with feedback of only a single phase, and that improvement is retained with quantized feedback.

The organization of this paper is as follows. Section II provides a brief review of the structure of extended orthogonal STBCs along with the proposed transmit antenna phase rotation scheme based on feedback. The simplifications for the first method are provided in Section III. We present simulation results in section IV for a multiple transmit and one receive antenna system comparing the case of the proposed exact channel phase feedback with previous work. We also consider the more practical case where the channel phase is quantized (using a uniform scalar quantizer) and compare the result with the ideal case of infinite precision as well as the previous work. We conclude in section V.

\section{EXTENDED ORTHOGONAL STBCS}

\section{A. EO-STBCs for four transmit antennas}

In [2], it has been shown that full diversity and full code rate complex valued space-time block codes exist only for a dimension of two. 


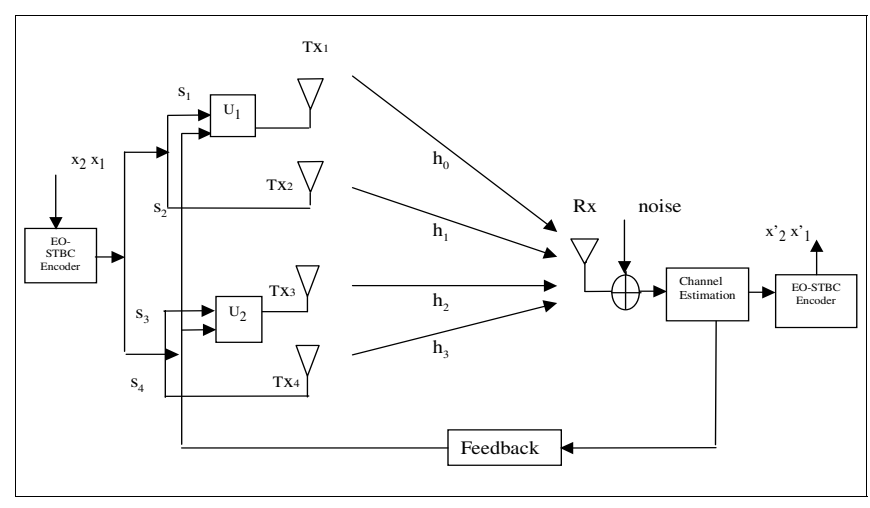

Fig. 1. Baseband representation of the proposed closed-loop EO-STBC system with 4 transmit and 1 receive antennas.

An example is the well-known Alamouti's scheme [1], which has the coding matrix:

$$
C_{(2)}=\left[\begin{array}{cc}
x_{1} & x_{2} \\
-x_{2}^{*} & x_{1}^{*}
\end{array}\right]
$$

where $x_{i}, i=1,2$ are complex symbols and (.)* denotes the complex conjugate operation. It is readily apparent that this is a rate one code. It takes two time-slots to transmit two symbols. More importantly, $C_{(2)}$ is a scaled unitary matrix which facilitates simple decoding. This scheme can be used as a building block to design the EO-STBC scheme with four transmit antennas and full rate. The scheme can achieve full transmit diversity with simple detection.

An example reported by Akhtar and Gesbert of extendedorthogonal STBCs designed in [7] is:

$$
E D_{(2)}=\xi\left[C_{(2)} C_{(2)}\right]=\xi\left[\begin{array}{cccc}
x_{1} & x_{2} & x_{1} & x_{2} \\
-x_{2}^{*} & x_{1}^{*} & x_{2}^{*} & x_{1}^{*}
\end{array}\right]
$$

Similarly, in [8], such a matrix was designed for a fourtransmit antenna system and has the coding matrix:

$$
E C_{(2)}=\xi\left[\begin{array}{cccc}
x_{1} & x_{1} & x_{2} & x_{2} \\
-x_{2}^{*} & -x_{2}^{*} & x_{1}^{*} & x_{1}^{*}
\end{array}\right]
$$

These two codes have the same performance. The parameter $\xi$ is a constant value equal to $1 / 2$, which for simplicity this constant will be ignored.

The equivalent channel matrix corresponding to the code in (3) used over four transmitters is given by:

$$
H_{(2)}=\left[\begin{array}{cc}
h_{0}+h_{1} & h_{2}+h_{3} \\
h_{2}^{*}+h_{3}^{*} & -h_{0}^{*}-h_{1}^{*}
\end{array}\right]
$$

where $h_{i}, i=0,1,2,3$ are the channel coefficients modelled by independent zero-mean complex Gaussian random variables with 0.5 variance per dimension.

Applying channel matched filtering, where $(.)^{H}$ denotes Hermitian transpose, we obtain [7]

$$
\Delta_{(2)}=H_{(2)}^{H} H_{(2)}=\left[\begin{array}{ll}
\alpha & 0 \\
0 & \alpha
\end{array}\right]
$$

with

$$
\alpha=\sum_{i=0}^{3}\left|h_{i}\right|^{2}+\beta
$$

where the interference factor

$$
\beta=2 \operatorname{Re}\left\{\left(h_{0} h_{1}^{*}+h_{2} h_{3}^{*}\right)\right\}
$$

and $|.|^{2}$ denotes the modulus squared of a complex number and $R e\{$.$\} its real part. It can be shown that the SNR is$

$$
S N R=\left(\sum_{i=0}^{3}\left|h_{i}\right|^{2}+\beta\right) \frac{\sigma_{x}^{2}}{\sigma_{n}^{2}}
$$

where $\sigma_{x}^{2}$ is the total transmit power of the desired signal, and $\sigma_{n}^{2}$ is the noise power at the receiver. It can be seen that the $\beta$ term may reduce the signal-to-noise ratio (SNR). In order to achieve a full diversity, we propose a feedback scheme to modify the transmitted signals from certain antennas by rotating with an appropriate phase angle to ensure that the $\beta$ term is maximized during the transmission period.

\section{B. CLOSED-LOOP EO-STBCS}

The feedback-based STBC for the enhancement of a diversity system with four transmit and one receive antennas system is shown in Figure 1. In [8], for a four antenna scheme, it was proposed in order to achieve full diversity, that the transmitted signals $s_{1}$ and $s_{3}$ are multiplied by $U_{1}=(-1)^{i}$ and $U_{2}=(-1)^{k}$, where $i, k=0,1$, before they are transmitted from the first and third antennas, respectively; which means using 0 or $\pi$ as the rotation angle for the signals. Likewise in [7], the receiver computes the "interference factor" $\beta$ and feeds back +1 if $\beta \geq 0$ or -1 if $\beta<0$. In our proposed scheme, the data signals transmitted from the first and third antennas are instead rotated by phase angles(phase shifted) $U_{1}=e^{j \theta_{1}}$ and $U_{2}=e^{j \theta_{2}}$ respectively while the other two are kept unchanged. The phase element $e^{j \theta_{k}},(k=1,2)$ will introduce a time delay which as in beamforming [9] will steer the energy in a certain direction which gives directivity and thereby chooses the direction that matches the channel. Therefore, the error performance is improved at the expense of increased feedback overhead.

The phase rotation on transmitted symbols is importantly effectively equivalent to rotating the phases of the corresponding channel coefficients.

The received signals $r_{1}$ and $r_{2}$, conjugated for convenience, at two independent time intervals are expressed as follows

$$
\left[\begin{array}{c}
r_{1} \\
r_{2}^{*}
\end{array}\right]=\underbrace{\left[\begin{array}{cc}
U_{1} h_{0}+h_{1} & U_{2} h_{2}+h_{3} \\
\left(U_{2} h_{2}\right)^{*}+h_{3}^{*} & -\left(U_{1} h_{0}+h_{1}\right)^{*}
\end{array}\right]}_{\tilde{H}} \underbrace{\left[\begin{array}{c}
x_{1} \\
x_{2}
\end{array}\right]}_{X}+\underbrace{\left[\begin{array}{c}
n_{1} \\
n_{2}^{*}
\end{array}\right]}_{\tilde{N}}
$$


The decision vector $\tilde{X}=\left[\tilde{x}_{1}, \tilde{x}_{2}\right]^{T}$ with the receive vector $R=\left[r_{1}, r_{2}^{*}\right]$ can be calculated as

$$
\begin{gathered}
\tilde{X}=\tilde{H}_{(2)}^{H} R=\left(\left|U_{1}\right|^{2}\left|h_{0}\right|^{2}+\left|h_{1}\right|^{2}+\left|U_{2}\right|^{2}\left|h_{2}\right|^{2}+\left|h_{3}\right|^{2}\right. \\
\left.+2 \operatorname{Re}\left(U_{1} h_{0} h_{1}^{*}\right)+2 \operatorname{Re}\left(U_{2} h_{2} h_{3}^{*}\right)\right) X+V
\end{gathered}
$$

where $V=\tilde{H}_{(2)}^{H} \tilde{N}$ is a noise component. Since $\left|U_{1}\right|^{2}=$ $\left|U_{2}\right|^{2}=1$, then the channel gain, $g$ is

$$
g=\sum_{i=0}^{3}\left|h_{i}\right|^{2}+2 \operatorname{Re}\left\{\left(h_{0} h_{1}^{*} e^{j \theta_{1}}+h_{2} h_{3}^{*} e^{j \theta_{2}}\right)\right\}
$$

where $\theta_{k}, k=1,2$ are obtained by maximizing $\beta$, and correspondingly the SNR, as follows:

$$
\begin{aligned}
& \theta_{1}=-\operatorname{angle}\left(h_{0} h_{1}^{*}\right) \\
& \theta_{2}=-\operatorname{angle}\left(h_{2} h_{3}^{*}\right)
\end{aligned}
$$

\section{Common Phase-Rotation}

If $\theta_{1}=\theta_{2}$, a common phasor $e^{j \theta}$ can be used to rotate the transmitted symbols from the first and third antennas and the rotation angle is selected from a range between 0 and $2 \pi$. It is apparent that this does not change the transmitted power. Since the phase rotation on the transmitted symbols is effectively equivalent to rotating the phases of the corresponding channel coefficients, the new channel gain can be written as

$$
g=\sum_{i=0}^{3}\left|h_{i}\right|^{2}+2 \operatorname{Re}\left\{\left(h_{0} h_{1}^{*}+h_{2} h_{3}^{*}\right) e^{j \theta}\right\}
$$

In this case, the transmitter needs to have knowledge of a single phase. Therefore, $\theta$ is determined as

$$
\theta=-\operatorname{angle}\left(h_{0} h_{1}^{*}+h_{2} h_{3}^{*}\right)
$$

\section{REDUCTION OF FEEDBACK}

Due to practical limitations, the number of feedback bits required from the receiver to the transmitter should be kept as small as possible. In the sequel, we provide a method to reduce the number of feedback bits while retaining satisfactory forward link performance.

\section{Quantization}

Feeding back the exact value of the phase angle with, for example, fixed or floating point resolution requires very large feedback overhead. In practical application this may not be possible due to the very limited feedback bandwidth. Therefore, the phase angles should be quantized, and then these levels are fed back to the transmitter. Suppose, for each phase angle, if we have two bit feedback, then we can feedback only four phase level angles such that the phase angles are from the set of $\left\{\Theta_{1}, \Theta_{2}\right\} \in \Omega=[0, \pi / 2, \pi$ or $3 \pi / 2]$ then for the first antenna phase adjustment, the discrete feedback information corresponding to the phases may be selected according to

$$
\Theta_{1}=\arg \max _{\theta_{1} \in \Omega} \operatorname{Re}\left\{\left(h_{0} h_{1}^{*}\right) e^{j \theta_{1}}\right\}
$$

Similarly, for the third antenna phase adjustment, the phases may be selected according to

$$
\Theta_{2}=\arg \max _{\theta_{2} \in \Omega} \operatorname{Re}\left\{\left(h_{2} h_{3}^{*}\right) e^{j \theta_{2}}\right\}
$$

In this case the particular selection giving the largest values of (14) and (15) may be preferable, as it would provide the largest array gain and achieve full diversity advantage. This idea can also easily be applied to (13). We now make an observation that when phase angle is not quantized and when both the first and third antenna signals are rotated using the phase angles, this scheme can be considered as a combined beamformer and STBC [10], where the pairs of first and second antennas and third and fourth antennas are used as beamformers. Moreover, when the phase angles are quantized to only two levels $(0$ or $\pi)$, this scheme is reduced to the scheme proposed in [7]. Hence, the proposed scheme is expected to perform between the scheme in [7] and combined beamformer and STBC.

\section{SIMULATION RESULTS}

In Figure 2, we show BER performance of the proposed closed-loop EO-STBC for four transmit antennas. Only one antenna at the receiver was considered, but this technique can be extended to multiple receive antennas. The $\mathrm{x}$-axis shows the SNR in $\mathrm{dB}$ and the $\mathrm{y}$-axis shows the BER. In order to evaluate the BER performance, we considered quasi-static flat fading channels. The BER performance of the proposed phase angle feedback scheme was compared with the previous closed-loop EO-STBC using quadrature phase-shift keying constellation (QPSK) symbols. The results depicted in figure 2 demonstrate that the phase rotation without quantization method significantly improves the BER performance over the previous closed-loop EO-STBC scheme. At a symbol error probability of $10^{-3}$, the proposed scheme provides about one $\mathrm{dB}$ improvement. The figure also provides a comparison of the proposed scheme with closed-loop QO-STBC [4], where we clearly notice that the proposed scheme outperforms closed-loop QO-STBC. This is because in QO-STBC there is only diversity gain and no array gain, but in the proposed closed loop EO-STBC there is both diversity gain of order four and array gain. While both diversity gain and array gain improve systems performance (decrease error rate), the nature of these gains are very different. Diversity gain manifests itself in increasing the magnitude of the asymptotic slope of the BER curve, while array gain shifts the error rate curve to the left.

The simulation result of a practical scenario of a quantization method is depicted in figure 3 . The performance of the quantized method is very close to the performance of the un-quantized scheme (ideal phase rotation method), 


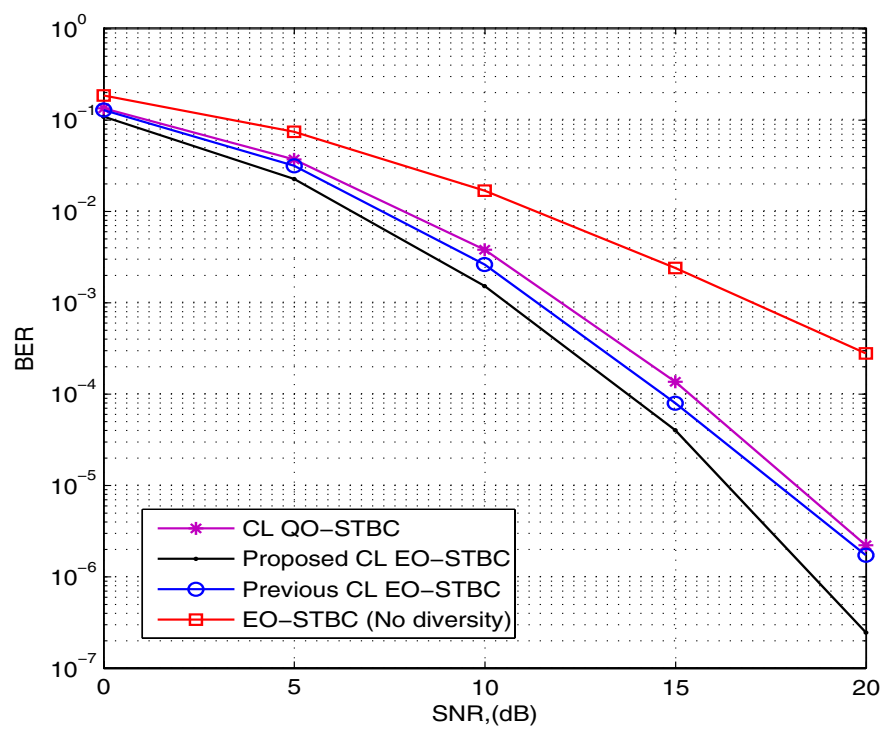

Fig. 2. Comparison of BER performance of the new scheme using ideal feedback channel information (infinite resolution) with four transmit and one receive antennas.

but still significantly better than that of the previous closed loop scheme. For the quantized method, we can see that the proposed scheme outperforms the previous closed loop EO-STBC with $0.75 \mathrm{~dB}$ at $\mathrm{BER}=10^{-3}$.

\section{Conclusions}

In this paper, we proposed a closed loop phase feedback method for EO-STBCs for enhancement of diversity. The principle is effective when ideal feedback (infinite resolution) is considered as shown in this paper. To include a more practical scenario, we also studied the effect of quantization and reduced the number of phases, performance gain was still attained. Finally, it is worth pointing out that simulation results show that the proposed closed-loop EO-STBC can outperform previous closed-loop STBCs for more than two transmit antennas.

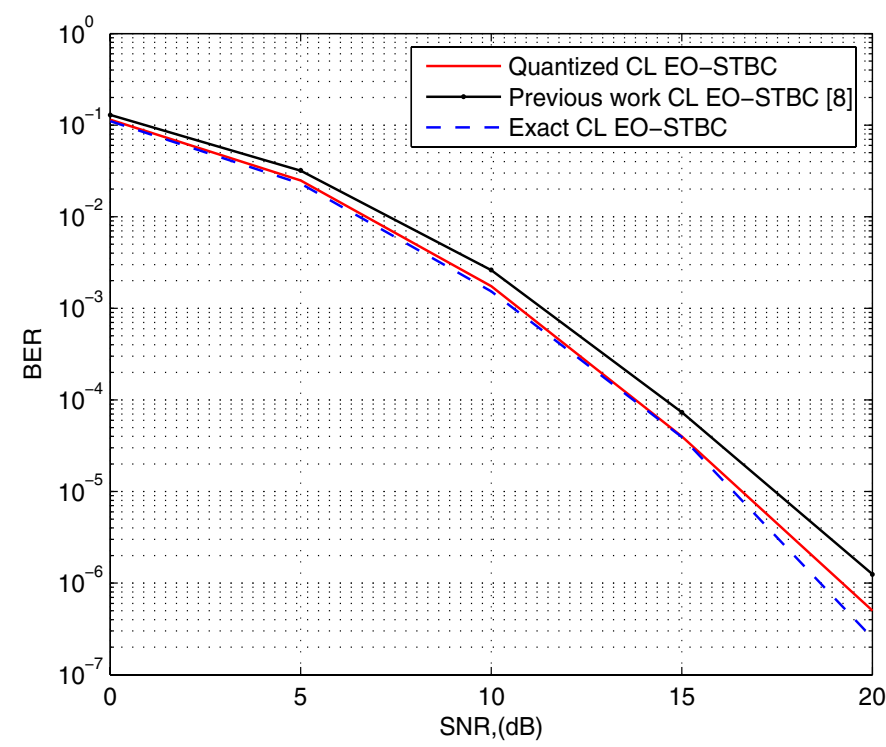

Fig. 3. Comparison of BER performance of the new scheme using quantized channel information with four transmit and one receive antennas.

\section{REFERENCES}

[1] S. M. Alamouti, "A simple transmit diversity technique for wireless communications," IEEE J.Select.Areas Commun., vol. 16, pp.1451 1458, Oct. 1998.

[2] V. Tarokh, H. Jafarkhani, and A. R. Calderbank, "Space time block codes from orthogonal designs," IEEE Trans. on Information Theory, vol.45, pp.1456-1467, July 1999.

[3] H. Jafarkhani, "A Quasi-Orthogonal Space-Time Block Code," IEEE Trans. Commun., vol. 49, No.1 pp.1-4, Jan. 2001.

[4] C. Toker, S. Lambotharan, and J. A. Chambers, "Closed-Loop QuasiOrthogonal STBCs and their Performance in Multipath Fading Environments and When Combined With Turbo Codes," IEEE Trans. Wireless Communications, Vol. 3(6), pp. 1890-1896, Nov. 2004.

[5] C. Toker, S. Lambotharan, and J. A. Chambers, "Space Time Block Coding for Four Transmit Antennas with Closed Loop Feedback over Frequency Selective Fading Channel," IEEE Information Theory Workshop, Paris, France, March/April 2003.

[6] S. Lambotharan, and C. Toker, "Closed-loop space time block coding techniques for OFDM based broadband wireless access systems," IEEE Trans. Consumer Electronics, vol. 51, pp. 765-769, August 2005.

[7] J. Akhtar and D. Gesbert, "Extended Orthogonal Block Codes With Partial Feedback," IEEE Trans. Wireless Commun., vol.3, No.6, pp. 1959-1962, Nov. 2004.

[8] Y. Yu, S. Keroueden, and J. Yuan, "Closed-Loop Extended Orthogonal Space-Time Block Codes for Three and Four Transmit Antennas," IEEE Signal Processing letters, Vol. 13, No.5, pp. 273-276, May 2006.

[9] G. Jongren, M. Skoglund, and B. Ottersten, "Combining beamforming and orthogonal space-time block coding," IEEE Trans. Inf. Theory, vol. 48, pp. 611-627, Mar. 2002.

[10] L. Liu, and H. Jafarkhani,"Application of Quasi-Orthogonal SpaceTime Block Codes in Beamforming," IEEE Trans. on Signal Processing, vol. 53, Jan. 2005.

[11] W. Su, and X. G. Xia,"Signal Constellations for Quasi-Orthogonal Space-Time Block Codes With Full Diversity," IEEE Trans. Inf. Theory, vol. 50, no. 10, pp. 2331-2347, Oct. 2004.

[12] J. K. Milleth, K. Giridhar, and D. Jalihal,"Closed-loop transmit diversity schemes for five and six transmit antennas," IEEE Signal Processing Letters, vol.12(2), pp.130-133, Feb. 2005. 